\title{
Can conservation targets for imperilled freshwater fishes and mussels be achieved by captive breeding and release programs? A systematic map protocol to determine available evidence
}

Lisa A. Donaldson ${ }^{1,2^{*}}$, Trina Rytwinski ${ }^{1,2,3}$, Jessica J. Taylor ${ }^{1,2}$, Joseph R. Bennett ${ }^{1,3}$, D. Andrew R. Drake ${ }^{4}$, André Martel ${ }^{5}$ and Steven J. Cooke ${ }^{1,2}$

\begin{abstract}
Background: Captive breeding programs are one of the many tools used by conservation practitioners as a means of conserving, supporting, and supplementing populations of imperilled species. Captive breeding programs exist around the globe for freshwater mussels and fishes, but the availability of evidence exploring the effectiveness of these programs has not yet been explored using systematic map criteria. This systematic map aims to identify, collate and describe the evidence that exists on the effectiveness of captive breeding programs, for the purpose of achieving conservation targets for imperilled freshwater fishes and mussels in the wild. The outputs of this systematic map will help to inform conservation managers and policy makers who are responsible for protecting imperilled freshwater species by identifying existing information and highlighting key information gaps for captive breeding programs operating in temperate regions.

Methods: This systematic map will search for, compile, and map existing literature on the effectiveness of captive breeding programs for the conservation of imperilled freshwater fishes and mussels. The systematic map will search using five bibliographic databases, two public search engines, and 19 specialist websites and will include both primary and grey literature. All studies that discuss details related to captive breeding programs for the conservation of imperilled freshwater fishes and/or mussels in temperate regions will be included in the map. The systematic map will produce a narrative report describing the evidence, including knowledge gaps evidence clusters, and a MS-Excel searchable database of articles and extracted metadata.
\end{abstract}

Keywords: Species at risk, Supportive breeding program, Recovery initiative, Hatchery, Propagation, Systematic map, Evidence synthesis, Conservation

\footnotetext{
*Correspondence: Lisa.Donaldson@carleton.ca

${ }^{1}$ Canadian Centre for Evidence-Based Conservation and Environmental Management, Institute of Environmental and Interdisciplinary Science, Carleton University, 1125 Colonel By Drive, Ottawa, ON, Canada

Full list of author information is available at the end of the article
} (http://creativecommons.org/licenses/by/4.0/), which permits unrestricted use, distribution, and reproduction in any medium, provided you give appropriate credit to the original author(s) and the source, provide a link to the Creative Commons license, and indicate if changes were made. The Creative Commons Public Domain Dedication waiver (http://creativecommons.org/ publicdomain/zero/1.0/) applies to the data made available in this article, unless otherwise stated. 


\section{Background}

Threats to freshwater mussels and fishes are widespread and often include habitat destruction, pollution, harvest, the introduction of disease, climate change (i.e., temperature increasing beyond species-specific thermal thresholds), and invasive species, to name a few $[1,2]$. Channelization, dam construction, and habitat fragmentation are also recognized as a significant contributor to the decline of freshwater fishes and mussels, particularly when countries lack legislative protection of essential habitat [3]. Freshwater mussels are critically important in freshwater ecosystems by providing a direct link between benthic and pelagic food webs, thereby contributing to nutrient cycling, stabilizing substrates, and providing habitat heterogeneity for other benthic species [4-7]. They also have important cultural value to Indigenous communities around the world. Despite their critical role, on a global scale, the freshwater mussel fauna is estimated to have among the highest rates of extinction and imperilment rates of any other group of organisms on Earth [3, 8, 9]. In fact, in the United States of America (USA) alone, $10 \%$ of freshwater mussels are classified as extinct, and $28 \%$ are federally listed as imperilled species (independent assessments estimate it to be upwards of 65\%) [10]. Similarly, freshwater fishes also play important roles in freshwater ecosystems, yet they have one of the highest extinction rates worldwide among vertebrates [10].

In recent years, captive breeding programs have increasingly been identified in federal recovery strategies and action plans to complement existing recovery measures and achieve recovery objectives for Species at Risk (SARA) listed freshwater fishes and mussels in Canada. Captive breeding programs are typically recommended in conjunction with other recovery measures, such as habitat restoration efforts and other activities to reduce threats to species in the wild (e.g., bycatch mitigation, changes to fishery regulations, changes to environmental conditions [11]). Fisheries and Oceans Canada has indicated that captive breeding programs should be considered in periods of very low survival when management interventions can be planned to augment the low survival, or when environmental conditions associated with low survival are predicted to change [12]. Captive breeding programs, by definition, breed animals in captivity under controlled environments to establish a small stock of labbred individuals, and are usually conducted to achieve one of three overarching goals, each with different quantifiable measures of success: (1) supplementing an existing population (i.e., bolstering small or declining populations in the wild to decrease the likelihood of local extirpation); (2) re-establishing a population where local extirpation has occurred; or (3) establishing an Ark population (e.g., zoo or hatchery stock) to ensure that a safe haven for genetic material and propagules exists. The objectives of the captive breeding programs are often focused on conservation of imperilled populations, but captive breeding may also overlap with fishery enhancement activities to create economic opportunities (generally referred to as "hatchery augmentation programs" for sport or commercial species; [13]). In general, the overall objectives of conservationoriented captive breeding programs are to reduce the risk of extinction and local extirpation, produce individuals that are genetically and phenotypically similar to wild populations, and increase the total number of individuals reproducing successfully in the wild.

Captive breeding programs are often very complex and have many different stages, including broodstock collection (i.e., the collection of individuals or gametes from the wild to be used for breeding purposes in captivity), spawning of reproductively prepared individuals (this can occur naturally in captivity or by human intervention), rearing of juveniles (involving very complex and often unknown rearing condition requirements), releasing individuals to the wild, and monitoring the success of the released individuals. Evidence from North America and Europe indicate that captive breeding programs have achieved numerous conservation successes; however, there are also documented negative consequences of captive breeding programs at both the individual and population level, including impacts on the donor populations. These issues confirm the importance of exploring captive breeding programs using systematic mapping criteria. In general, negative consequences can occur through:

1. Capturing of individuals from the wild for use as broodstock-unintentional selection of non-representative phenotypic characteristics (e.g., body size, sex ratios, life history strategy).

2. Raising individuals in captivity-domestication selection (from diets, method of obtaining food), behavioural adaptations (associated with rearing densities, social behaviours, risk taking, aggression), individual variability (growth rates, disease, fecundity), abiotic factors (water temperature, $\mathrm{pH}$, velocity, photoperiod, salinity), and human-interventions (vaccinations, antibiotics, disease treatments).

3. Release of individuals back into the wild-individual survival success (predation, competition, disease), ability for the natural environment to support the population, remediation of the source of the original cause of the decline, reduction of genetic variation in 
the population, mixing of genetic stocks, inadvertent transfer of disease, and many more [14].

Despite these considerations, captive breeding programs offer tremendous promise for the conservation of imperilled species around the globe. Understanding what evidence is available to assess the effectiveness of these programs for their ability to achieve conservation targets in the wild will help to support management decisions and the recovery of aquatic species at risk. Specifically, it will help to identify the information available for specific species to maximize the probability of success, minimize costs, maximize public benefits and maximize individuals available for reintroduction efforts [15].

\section{Stakeholder engagement}

This systematic map was initiated by the Canadian Centre for Evidence-Based Conservation and Environmental Management (CEBCEM) to provide support to the Canadian Freshwater Species at Risk Research Network (2017-2020), led by Fisheries and Oceans Canada (DFO). The network is a national academic research network that has a primary goal of addressing priority research questions for imperilled freshwater fishes and mussels in Canada. The network has two theme areas, including (A) identifying threat mechanisms, and (B) conducting research to support species reintroductions. This systematic map will provide resource managers and scientists with a collated summary of existing information on the effectiveness of captive breeding programs at achieving conservation targets in the wild for imperilled freshwater fishes and mussels in temperature regions. It is commonly believed that there is currently a paucity of knowledge related to the effectiveness of these programs (e.g., [16]), especially for freshwater mussels, but the state of knowledge on this topic has not yet been evaluated by systematic mapping procedures and there exists potentially great benefit to the global community in doing so. One area in particular that would benefit greatly from a better understanding of the existing knowledge state is culturing practices for imperilled species. The complexity of life histories of freshwater mussels and fishes, and, in some cases, the inability to successfully rear species in captivity, leads to much of this research being exploratory in nature. In many cases, these species have not been traditionally cultured, so husbandry techniques are not well established and need to be learned and developed. Also, much of this work is evolving where culture methods change rapidly to account for new species, threats, and environmental influences. As such, much of the information associated with measuring the success of these programs is available as grey literature. This information is often difficult to obtain and collate but offers an incredible wealth of knowledge and information from practitioners with hands-on experience under a variety of conditions.

During the formulation of the systematic map question, an Advisory Team composed of ten stakeholders and scientific experts was established and consulted. The Advisory Team consists of conservation experts from Canada, the USA and Germany having research experience related to freshwater fishes and/or mussels, including expertise on genetic conservation and captive breeding programs. The titles of some of the Advisory Team members include research scientist, professor, conservation science and education researcher, biodiversity conservation policy advisor, and research biologist. Their affiliations include government (Fisheries and Oceans Canada, Ontario Ministry of Natural Resources and Forestry, Canadian Museum of Nature), academic (Carleton University, Technical University of Munich) and non-governmental organization (NGO; Tennessee Aquarium). The role of the Advisory Team is crucial to all aspects of this systematic map, through: project initiation, primary question formulation, suggestions of search terms to use, generation of the benchmark list, suggestions of specialist websites to search, and metadata extraction strategy. The Advisory Team will continue to participate in this systematic map through to completion and will be invaluable for helping to acquire grey literature on this subject. It is anticipated that a significant amount of grey literature exists on this topic, including in government and private facilities, and the network of people and organizations that the Advisory Team helps the Review Team access is central to the success of this systematic map. The Review Team and Advisory Teams will continue to connect as a group via regularly-scheduled teleconferences and email updates.

\section{Objective of the review}

The objective of this systematic map is to identify, collate and describe the information that exists on the effectiveness of captive breeding programs, for the purpose of achieving conservation targets for imperilled freshwater fishes and mussels in the wild in temperate regions. The systematic map will produce a report describing evidence availability, clusters and knowledge gaps. A searchable MS-Excel database of articles and extracted metadata will also be included as additional documents with the final published systematic map.

\section{Primary question}

What evidence exists on the effectiveness of captive breeding and release programs for imperilled freshwater fishes and mussels in temperate regions? 


\section{Components of the primary question}

The project is relevant to imperilled freshwater fish and mussel species, defined as those with conservation status in their relevant jurisdiction, in temperate regions around the world. In Canada, this includes species assessed by the Committee on the Status of Endangered Wildlife in Canada (COSEWIC) as extirpated, endangered, threatened, and of special concern, as well as those listed under the Species at Risk Act (SARA), plus relevant provincial and territorial assessments. In other countries, species inclusion will be based on the relevant conservation listing process (e.g., [17], federal, state-level, and international including IUCN Red List).

\section{Population}

Imperilled freshwater fishes and mussels that have captive breeding programs occurring in temperate regions around the globe. This includes all fishes and mussels that have at least one stage of their lives in freshwater, including both migratory and non-migratory species. Species that only live in the marine environment are not included in this systematic map. Imperilment status of freshwater fish and mussel species is defined as those with any conservation status in their relevant jurisdiction.

\section{Intervention/exposure}

Captive breeding programs. A captive breeding program can be defined in a number of ways and includes a number of objectives and successive steps including (but not limited to): supplementing an existing population, re-establishing a population where local extirpation has occurred, reintroduction and/or establishing an Ark population. For this systematic map, the purpose of the captive breeding program should be for conservation management objectives, not explicitly for the enhancement of sport fishing populations to create economic opportunities (generally referred to as "hatchery augmentation programs; [11]) or for supplementation of commercial fisheries. All stages of the captive breeding program will be included in this systematic map, including articles related to the collection of broodstock, rearing and release methods, and monitoring of the released individuals in the wild. Articles that do not explicitly discuss details of an existing captive breeding program, and instead focus only on the current status of a population in the wild and the potential for the establishment of a new program, will be excluded from the systematic map.

\section{Comparator}

Wild individuals and/or populations of the same species within the same geographical area or close proximity.
Individuals within the same captive breeding program that experience different conditions (e.g., water temperatures, release methods).

\section{Outcomes}

Generally, the outcomes of captive breeding programs can be measured in broad categories, including changes in genetic diversity (e.g., what is the difference in genetic diversity within a population before and after supplementation by captive bred individuals?), changes in abundance of populations (e.g., what is the difference in population size before and after supplementation by captive bred individuals?), and distribution in the wild (e.g., what is the difference in the local population distribution before and after supplementation by captive bred individuals?). All outcomes will be included and mapped in this systematic map, as understanding what these outcome variables are is valuable (and often unknown) information that can contribute to the overall understanding of the state of literature on this topic.

\section{Methods}

The review will follow the Collaboration for Environmental Evidence Guidelines and Standards for Evidence Synthesis in Environmental Management [18] and conform to ROSES reporting standards [19] (see Additional file 1).

\section{Searching for articles}

Included in the systematic map will be primary literature in peer-reviewed journals and grey literature (e.g., theses, government papers, organisation reports, consultant reports). Digital media will be screened, when they are available online without the need for purchasing the media or having specialized pay-for-use software to view it. The Interlibrary Loans program at Carleton University will be used to acquire hard or digital, full-text copies of any articles that are included once the title and abstract screening has occurred. All bibliographic databases will be accessed using Carleton University's institutional subscriptions as outlined in Additional file 2. All searches will be completed by one Reviewer using the web browser Safari and it will be placed into "private mode", to reduce user-specific search results. When a complex search string is not accepted by the search engine, the help menu will be consulted, and the search terms will be modified.

\section{Search terms and languages}

The Review Team generated a list of potentially relevant search terms and benchmark articles (as outlined in Additional file 3) through a scoping exercise done 
in collaboration with the Advisory Team. The titles, abstract, keywords and reference lists of each of the benchmark article were read and words that were often used in the literature on the topic were added to the preliminary list of search terms. The search terms were categorized into terms related to the population, intervention, and outcome as defined by the 2018 Collaboration for Environmental Evidence (CEE) guidelines [18]. The terms in each of the categories of population, intervention and outcome can be combined using the Boolean operator "OR", as suggested in the search string. The asterisk (*) is a wildcard and represents any characters (e.g., conserv* includes conservation, conserved, conserving). The categories are then combined using "AND" in the following general format: (population) AND (intervention substring) AND (outcome substring).

The focus of this systematic map is to collate existing information on captive breeding programs for achieving conservation targets for imperilled freshwater fishes and mussels in the wild. There has been much discussion with the Advisory Team about how/if 'at risk or imperilled' (or synonyms of) should be included in the search terms. The Review Team suggested that the Canadian government's Species at Risk Public Registry be used to generate a list of known imperilled species [20]. The Species at Risk Public Registry was searched, and the following filters were applied to the list of species: taxonomy group [molluscs, fishes (freshwater)],
[Committee on the Status of Endangered Wildlife in Canada (COSEWIC) risk category (extirpated, endangered, threatened, special concern, data deficient)]. No geographical restrictions were applied (i.e., species could be from anywhere in Canada). The resulting species included all those in the phylum Mollusca. Those species were then further filtered to only those within the order Unionoida. Of all remaining species, the following SARA status was represented in the species list: extirpated, endangered, threatened, special concern and no status. It also included species that have been assessed under Schedule 1, Schedule 2, Schedule 3, or No Schedule (in instances where the population has been assessed by COSEWIC but has no SARA status). The search results were exported into MS-Excel and the scientific names of each species were collated and combined using the Boolean operator "OR". The rationale for this is that the words "fish" and "mussel" are not always included in the title, abstract and/or keywords of articles. Although the list of species included in the search was created based on Canadian criteria, and thus there exists the potential for the search results to be biased towards Canadian species, the species included live in temperate regions throughout the world. The terms are connected with "OR" Boolean operators, and "mussel* OR mollus" OR unionid* OR fish OR fishes" are included to search other species with conservation concern more broadly (Table 1).

No language restrictions will be placed on the search outputs from publication databases. All articles yielded

Table 1 Search string for execution of the search strategy (Access: Carleton University subscription)

\begin{tabular}{|c|c|}
\hline Component & Search string \\
\hline Population & 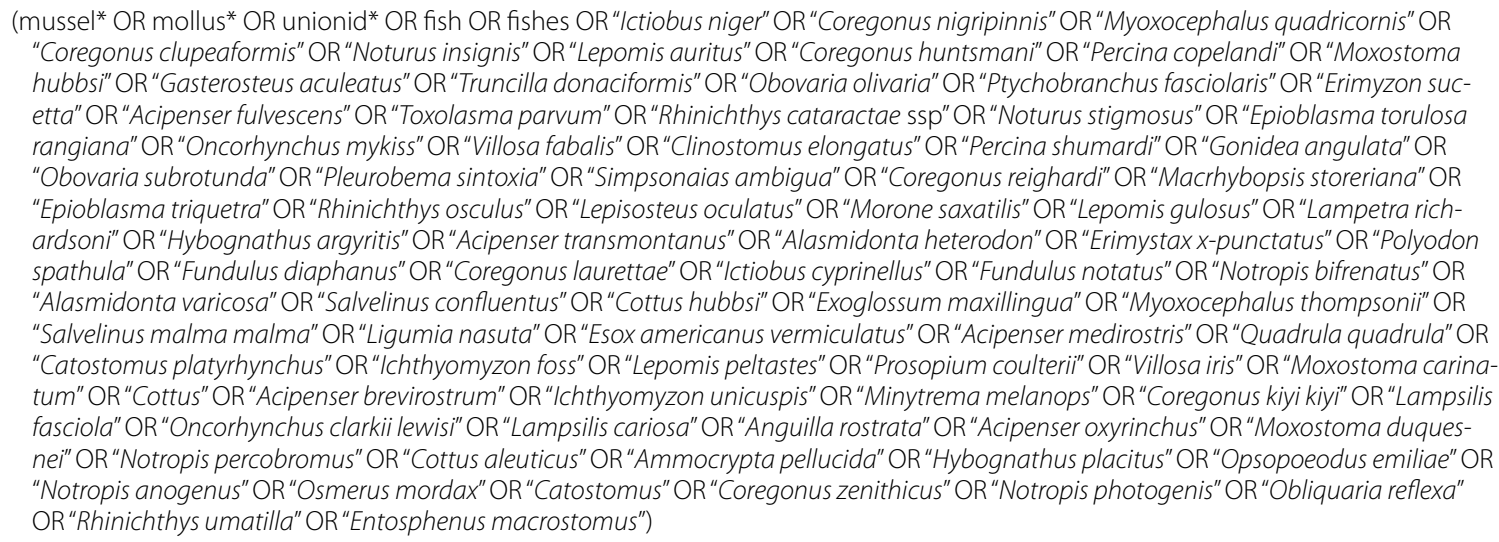 \\
\hline Intervention & $\begin{array}{l}\text { (captive OR "captive-bred" OR "captive breeding" OR "captive breeding program" OR "captive breeding programme" OR "captive reared" } \\
\text { OR "captive-rearing" OR coancestry OR cryopreservation OR cryopreserved OR enhancement OR hatcheries OR hatchery OR"live } \\
\text { gene bank" OR"live gene banking" OR "recovery initiative" OR "stock enhancement" OR supplementation OR "supportive-breeding" OR } \\
\text { "supportive breeding program" OR "supportive breeding programme" OR "supportive rearing") }\end{array}$ \\
\hline Outcomes & $\begin{array}{l}\text { (conservation OR conserv* OR biodiversity OR "biodiversity conservation" OR "biological diversity" OR recover* OR re-establish* OR } \\
\text { reintroduc* OR re-introduc* restor*) }\end{array}$ \\
\hline
\end{tabular}


from the search string will be exported from the databases and uploaded into EPPI-Reviewer 4 for article screening (available online [21]). The call for grey literature will be circulated in English only.

\section{Searching bibliographic databases}

All bibliographic databases will be accessed using Carleton University's institutional subscriptions as outlined in Additional file 2.

1. ISI Web of Science core collection-Multidisciplinary research topics including journals, books, proceedings, published data sets and patents.

2. Scopus-Abstract and citation database of peerreviewed literature including journals, books and conference proceedings.

3. ProQuest Dissertations and Theses Global-International depository of graduate dissertations and theses.

4. Federal Science Library-Canadian government books, reports, government documents, theses, conference proceedings and journal titles. This search platform is freely available online and does not require a subscription.

5. Science.gov-United States Federal Science. This search platform is freely available online and does not require a subscription.

\section{Web-based search engines}

1. Google

2. Google Scholar

Internet searches will be performed using the search engines Google and Google Scholar and the resulting references will be sorted by relevance. The first 500 citations will be screened for relevance at the level of title, then abstract and full text (when appropriate). If the reviewer notices that the level of relevance of each article significantly declines before screening 500 articles, the reviewer will stop when the relevance significant declines (as per suggestion by Livoreil et al. [22]).

Two search strings will be used in each of the search engines, one for fish and the other for mussel. This is because Google and Google Scholar do not allow for nested AND/OR statements. The search strings will be:

1. Fish breeding OR propagate OR stocking OR coancestry OR cryopreservation OR enhancement OR hatchery OR supplementation OR rearing -"fish oil".
2. Mussel breeding OR propagate OR stocking OR coancestry OR cryopreservation OR enhancement OR hatchery OR supplementation OR rearing.

Similar to database searches, customized search strings used in search engines (and specialist websites below) will be recorded and provided in an additional file in the systematic map. All resulting relevant articles will be included in the article database.

\section{Organisational websites}

Specialist organization websites listed below will be searched.

1. American Fisheries Society Imperilled Freshwater and Diadromous Fishes of North America

2. Atlantic Salmon Federation

3. Conservation Fisheries

4. Desert Fish Council

5. European Commission-Life Programme

6. Fisheries and Oceans Canada

7. Fisheries Research Service

8. Food and Agriculture Organization of the United Nations

9. Freshwater Mollusk Conservation Society

10. Land and Water Australia

11. Ministry of the Environment New Zealand

12. National Oceanic and Atmospheric Administration

13. Natural Resources Wales

14. North American Native Fishes Association

15. Ontario Ministry of Natural Resources and Forestry

16. Parks Canada

17. Trout Unlimited

18. United Nations Environment Programme

19. US Fish and Wildlife Service.

The search strings used will be as follows:
A. Fish breeding conservation
B. Mussel breeding conservation
C. Fish propagate conservation
D. Mussel propagate conservation

Page data from the first 20 search results for each search string will be extracted, screened for relevance, and searched for links or references to relevant publications, data and grey literature. The list of websites was narrowed to the following organizations after consulting with our Advisory Team for relevance. 


\section{Supplementary searches}

Reference sections of included articles will be hand searched to evaluate articles that have not been found using the search strategy. Authors of any unpublished references will be contacted to request access to the full article. Stakeholders will be consulted for insights and advice for new sources of information. The Review Team will contact authors of unobtainable articles in an attempt to gain access to the full article. A call for grey literature will be circulated via professional networks and professional association distribution lists to solicit articles for inclusion in this systematic map. The Review Team will also use social media and email to alert the community of this systematic map and to reach out to recognized experts and practitioners for further recommendations and for provision of relevant unpublished material.

\section{Estimating the comprehensiveness of the search}

A list of benchmark articles were compiled with feedback from the Advisory Team (Additional file 3). The search strategy was tested using the search string in Web of Science Core Collection (subscription access: Carleton University). Articles yielded by the search string were exported into a .CSV file format and opened in MS-Excel. The list was then cross-checked with the list of key references to see which articles were found and which were not. Articles that were not found were explored at full text to evaluate why they were not selected (e.g., governmental report not published in a journal contained in the Web of Science Core Collection, consulting report etc.). The comprehensive of the search string was assessed by determining the number of key reference articles that were found in the test searches of the search string. During the scoping exercise (completed between 29/11/2017 and $24 / 01 / 2018$ ), the above search string found 2403 articles, of those 10 of the 18 key articles listed were found (Additional file 3). Of the articles that were not found during the scoping exercise, three articles were Canadian federal government reports and that were then captured using the Federal Science Library database. Any updates made to the search string and search strategy as the systematic map is conducted will be recorded and all amendments will be reported in the final published document.

\section{Article screening and study eligibility criteria Screening process}

Articles found using the search strategy will be screened at two distinct stages, (1) title and abstract, and (2) full text. When English title and abstracts are found, with article content in other languages, the articles will be screened as part of the title and abstract screening process. If the article is included to full text screening, a translated version of the article will be searched online for (using Google Scholar) and, when translated copies are not found, the first author of the relevant article will be contacted to see if a translated copy exists and is available.

\section{Consistency checking}

Before the screening of title and abstracts begins, two reviewers using a random subset of $10 \%$ of all articles or 100 titles/abstracts (whichever is bigger) will undertake consistency checks to ensure consistent and repeatable decisions are being made in regard to which articles get screened out and which go on in the process to be further reviewed. The two reviewers will use a Kappa test to determine consistencies in screening decisions. The Kappa statistic tests the reliability of testers, essentially asking how many times reviewers choose the same outcome. A Kappa score of $\geq 0.6$ indicates substantial agreement between reviewers and will be required to be achieved before any further screening is conducted for the map. The results from the consistency check will be discussed and discrepancies will be reviewed by both reviewers to understand why the choice was made to include/exclude the article. The articles where included/ exclusion decisions were not the same between reviewers will be reviewed and screening will not continue until the decisions are reconciled. This same process will be repeated prior to screening articles at full text (i.e., two reviewers using a random subset of $10 \%$ of all articles that were included at title and abstract, will undertake consistency checks and a Kappa score of $\geq 0.6$ will be required before any further screening is conducted). Articles or datasets found by means other than database or search engine searches (i.e., specialist website or other literature searches) will be entered at the second stage of this screening process (i.e., full text) but will not be included in consistency checks. All article screening decisions will be included in the database, so it will be clear at what level any article was excluded. If the decision to include or exclude a specific article is unclear, that article will be retained and will go on to the next level of screening. If there is further doubt, the Review Team will discuss those articles as a group to make a decision. Any articles that do not have abstracts will automatically be screened at the full text level. A list of all articles excluded at the full text level will be provided as a additional file to the systematic map and will include the reason for exclusion. 


\section{Eligibility criteria}

The following eligibility criteria will be used to screen articles at title/abstract and full text screening.

\section{Eligible populations}

Freshwater fishes and mussels will be included in the systematic map, including anadromous, catadromous, and potadromous species. Marine-only and oceanodromous species will be excluded. All life stages of the animal will be included. Population of fish and/or mussel species must be in North $\left(23.5^{\circ} \mathrm{N}\right.$ to $\left.66.5^{\circ} \mathrm{N}\right)$ or South $\left(23.5^{\circ} \mathrm{S}\right.$ to $66.5^{\circ} \mathrm{S}$ ) temperate regions. Fishes and/or mussels must be identified in the article as 'at risk' in their respective jurisdictions (e.g., IUCN, federal, or state-level criteria).

\section{Eligible interventions}

Captive breeding program, or any component thereof, that have a conservation objective (i.e., not solely for the creation of sport fishing opportunities, often termed "hatchery augmentation"; or for commercial and/or aquaculture purposes for the food industry [11]). The intention of the captive breeding program can include:

1. Supplementation: The purpose of the captive breeding program should be supplementation as defined by Waples et al. [23] to be "the intentional demographic integration of hatchery and natural population, with the goal of improving the status of an existing natural population (either in an absolute sense or relative to what its status would be without supplementation)."

2. Reintroduction: The purpose of the captive breeding program should be "to establish a healthy, genetically diverse, self-sustaining population in an area where it has been extirpated" and includes the development of Ark populations [24].

\section{Eligible comparators}

Each of the different stages of the captive breeding program may have a different comparator type. Information presented on the population status before and after the onset of a captive breeding program (e.g., genetic diversity of population, population size) will be considered relevant, as will comparisons made between wild-born and captive-born individuals. When a comparator does not exist in an article (e.g., in the instance of reporting on rearing conditions of a particular imperilled species), the articles will be included in the systematic map but will be coded separately in the database to indicate a lack of comparator.

\section{Eligible outcomes}

Little is known about what the state of the knowledge is, and how captive breeding programs are evaluated. The purpose of this systematic map is to help identify the types and quantity of existing evidence related to the effectiveness of captive breeding programs for the conservation of imperilled freshwater fishes and mussel species. As such, any outcome metric related to evaluating the effectiveness of these programs will be included in this map. Some of the outcome metrics that are anticipated to be reported on are measures of genetic diversity, populations size, and distribution in the wild. Information on success/failure relative to intermediate stages of the program (e.g., ability to raise the organism in captivity, husbandry techniques) will also be collated and discussed.

\section{Eligible types of study design}

All study designs will be included in the systematic map and the type of study will be coded. It is anticipated that study designs could include before/after (BA), control/ impact (CI), as well as studies combining these types of comparisons, before/after/control/impact (BACI) and Randomized Controlled Trials (RCT). Post-treatment (PT) designs (i.e., a single post-treatment monitoring period, or a temporal correlation design using multiple post-treatment monitoring periods without true before data) and Impact only (I-only) designs (i.e., no control site, or a spatial correlation that does not include "zerocontrol" site(s)). Understanding what study design types exist in the literature for these types of programs will be insightful for understanding the potential for future quantitative analysis. Theoretical studies, review articles, and policy discussions will be excluded from the systematic map but their reference lists will be screened for relevant articles.

Date range No date restrictions will be applied. Articles from all years will be included.

\section{Reasons for exclusion}

Articles will be coded to identify on what criteria used for exclusion [(e.g., EXCLUDE (population), EXCLUDE (intervention), EXCLUDE (comparator), EXCLUDE (outcome)]. A list of articles excluded at full text level, along with the reason(s) for exclusion, will be provided as an additional file with the systematic map document. When multiple articles report on the same captive breeding program, the most recent article will be retained unless different metrics were reported for studying the effectiveness of the program, or the data were presented at a different level of aggregation (e.g., total or individual species mean values). This technique, following the protocols 
used by Sciberras et al. [25], aims to avoid the risk of double counting data.

\section{Study validity assessment}

No formal study validity assessment will be performed on included articles for this systematic map.

\section{Data coding strategy}

Metadata will be extracted from the included articles by the Review Team and will be recorded in a MS-Excel database that will be made available with the published systematic map. The extracted information will be used to identify trends, knowledge gaps and clusters in the evidence-base.

The information that will be also coded for each article includes (but is not limited to):

1. Bibliographic information-article citation, title, authors, article type, name of publisher, year

2. Population-Animal type (i.e., mussel, fish), scientific name, common name, family, population name, life history strategy (e.g., migratory, non-migratory).

3. Protection status-Protection classification (e.g., endangered, special concern, threatened, data deficient), governing organization (e.g., IUCN, SARA, COSEWIC, ESA), country of status origin.

4. Captive breeding program-Objective of the captive breeding program [e.g., propagation (PROP), reintroduction (REIN), supplementation (SUPP), experimental (EXP)], conservation target category, name of breeding facility, country facility is in, state/province name, name of program contact, contact information, year the program started.

5. Study component-What component of the captive breeding program is discussed in the article [collection of individuals from the wild (COLL), rearing of individuals (REAR), release of individuals (REAL), multiple stages (MUL)].

6. Study type-Study design (CI/BA/BACI/RCT, PT, I-only, none), type of control [wild population (WILD), captive population (CAPT), no control (NONE), other $(\mathrm{OTH})]$, source of individuals used in the program [wild population (WILD), captive population (CAPT)].

7. Data reporting-Data reporting type (quantitative, quantitative approximations, semi-quantitative, qualitative), methodological detail (low, medium, high).

8. Outcome reporting-Were results analysed statistically (yes, no, unclear), were power analysis conducted (yes, no, unclear), were confounding factors stated (yes, no), confounding factors (free text), outcome category [genetic diversity (GEN), abundance (ABUN), survival (SUR), recruitment (REC), growth
(GRO), behaviour (BEH), other $(\mathrm{OTH})]$, outcome metric [expected heterozygosity (HE), genetic diversity (GEN), hatching-percent (HAT \%), heterozygosity $(\mathrm{H})$, none reported (NR), number of alleles (NA), nucleotide distance (ND), number of individuals $(\mathrm{N})$, observed heterozygosity $(\mathrm{HO})$, other $(\mathrm{OTH}$, free text), survival-percent (SUR \%), survival-number of individuals (SUR\#), total length (TL), total number private alleles (NP), weight (W)], measures of variation [standard deviation (STDEV), standard error (STER), confidence intervals (CONI)], sample size.

Note that categories will be added iteratively as encountered. Metadata will be extracted from all included articles after all full-text screening is completed. The extracted information will be entered into a MS-Excel document. In instances of missing or unclear information, corresponding authors for each article will be contacted by email if time and resources allow.

\section{Consistency checking}

To ensure that information is being coded and extracted in a consistent and repeatable manner, two reviewers will extract information from five of the same articles (similar to methods used for consistency checking during the screening process). Afterwards, the information will be compared. Any inconsistencies will be discussed amongst the Review Team members, and if any disagreement occurs, they will be discussed with the entire Review Team.

\section{Demonstrating procedural independence}

If the instances occur where a Review Team member involved in this systematic map has authored an article that is being considered in the review, that reviewer will not be involved in screening the article (i.e., in the determination of whether the study should be included or excluded). Instead, a separate reviewer will independently look at that article to conduct all parts of the mapping process. It is only after data extraction is complete that the author of the article may be involved if clarity of information provided in the article is required.

\section{Study mapping and presentation}

There will be two main outputs from this systematic map, including a written narrative synthesis and searchable, coded database (MS-Excel). Descriptive statistics will be used to describe the overall amount (e.g., number of articles, number of studies) and subgroups (e.g., species, geographic locations, stages in the captive breeding program, outcome metrics) of evidence available. Key knowledge gaps (areas that are 
under-represented in the evidence base and could warrant further research) and knowledge clusters (areas of evidence that are well-represented and could potentially be good topics for future systematic reviews) will be identified using visual heat maps (MS-Excel). The narrative synthesis will aim to be as visual as possible, summarizing information in tables and figures. The ultimate goal of this systematic map is to identify, collate and describe the information that exists on captive breeding programs for imperilled freshwater fishes and mussels. Understanding what programs exists, the different components of these programs, and what outcome metrics are commonly reported in the literature will help guide further exploration on evaluating the effectiveness of these programs.

\section{Additional files}

Additional file 1. ROSES form for systematic map protocols.

Additional file 2. Institutional subscriptions.

Additional file 3. Benchmark list.

\section{Authors' contributions}

All authors read and approved the final manuscript.

\section{Author details}

${ }^{1}$ Canadian Centre for Evidence-Based Conservation and Environmental Management, Institute of Environmental and Interdisciplinary Science, Carleton University, 1125 Colonel By Drive, Ottawa, ON, Canada. ${ }^{2}$ Fish Ecology and Conservation Physiology Laboratory, Department of Biology, Carleton University, 1125 Colonel By Drive, Ottawa, ON, Canada. ${ }^{3}$ Department of Biology and Institute of Environmental and Interdisciplinary Science, Carleton University, 1125 Colonel By Drive, Ottawa, ON, Canada. ${ }^{4}$ Great Lakes Laboratory for Fisheries and Aquatic Science, Fisheries and Oceans Canada, 867 Lakeshore Road, Burlington, ON, Canada. ${ }^{5}$ Zoology, Research and Collections, Canadian Museum of Nature, 1740 Pink Road, Gatineau, Canada.

\section{Acknowledgements}

The authors would like to thank several reviewers and collaborators who provided valuable insights to strengthen this systematic map protocol including members of the Advisory Team: Alan Dextrase (Biodiversity Conservation Policy Advisor, Ontario Ministry of Natural Resources and Forestry, Peterborough, Ontario), Anna George (Vice President of Conservation Science and Education, Tennessee Aquarium, Chattanooga, Tennessee), Jürgen Geist (Professor, Aquatic Systems Biology Unit, Department of Ecology and Ecosystem Management, Technical University of Munich, Germany), Todd Morris (Research Scientist, Great Lakes Laboratory for Fisheries and Aquatic Sciences, Fisheries and Oceans Canada, Burlington, Ontario), and Patrick O'Reilly (Research Scientist, Salmon Population Geneticist, Fisheries and Oceans Canada, Dartmouth, Nova Scotia). Comments were also kindly provided by Teah Lizée, Shannon Clarke and Elizabeth Robson Gordon (Fish Ecology and Conservation Physiology Lab, Carleton University, Ottawa, Ontario).

\section{Competing interests}

The authors declare that they have no competing interests.

\section{Availability of data and materials}

Not applicable.
Consent for publications

Not applicable.

Ethics approval and consent to participate

Not applicable.

\section{Funding}

The study was primarily supported by Fisheries and Oceans Canada. Additional support is provided by the Natural Science and Engineering Research Council of Canada, The Canada Research Chairs Program, and Carleton University.

\section{Publisher's Note}

Springer Nature remains neutral with regard to jurisdictional claims in published maps and institutional affiliations.

Received: 16 August 2018 Accepted: 5 March 2019

Published online: 02 April 2019

\section{References}

1. Venter O, Brodeur NN, Nemiroff L, Belland B, Dolinsek IJ, Grant JW. Threats to endangered species in Canada. AIBS Bull. 2006;56(11):903-10.

2. Dextrase AJ, Mandrak NE. Impacts of alien invasive species on freshwater fauna at risk in Canada. Biol Invasions. 2006;8(1):13-24.

3. Haag WR, Williams JD. Biodiversity on the brink: an assessment of conservation strategies for North American freshwater mussels. Hydrobiologia. 2014;735(1):45-60.

4. Vaughn CC, Gido KB, Spooner DE. Ecosystem processes performed by unionid mussels in stream mesocosms: species roles and effects of abundance. Hydrobiologia. 2004;527(1):35-47.

5. Boeker C, Lueders T, Mueller M, Pander J, Geist J. Alteration of physicochemical and microbial properties in freshwater substrates by burrowing invertebrates. Limnol Ecol Manag Inland Waters. 2016;59:131-9.

6. Richter A, Stoeckl K, Denic M, Geist J. Association between the occurrence of the Thick-shelled River Mussel (Unio crassus) and macroinvertebrate, microbial, and diatom communities. Freshw Sci. 2016;35:922-33.

7. Lummer EM, Auerswald K, Geist J. Fine sediment as environmental stressor affecting freshwater mussel behavior and ecosystem services. Sci Total Environ. 2016;571:1340-8.

8. Geist J. Integrative freshwater ecology and biodiversity conservation. Ecol Ind. 2011;11:1507-16.

9. Lopes-Lima M, Sousa R, Geist J, Aldridge DC, Araujo R, Bergengren J, et al. Conservation status of freshwater mussels in Europe: state of the art and future challenges. Biol Rev. 2017:92:572-607.

10. Burkhead NM. Extinction rates in North American freshwater fishes, 1900-2010. Bioscience. 2012;62(9):798-808.

11. Naish KA, Taylor JE III, Levin PS, Quinn TP, Winton JR, Huppert D, et al. An evaluation of the effects of conservation and fishery enhancement hatcheries on wild populations of salmon. Adv Mar Biol. 2007;53:61-194.

12. Fisheries and Oceans Canada. Evaluation of captive breeding facilities in the context of their contribution to conservation of biodiversity. DFO Canadian Science Advisory Secretariat Science Advisory Report 2008/027. 2008.

13. Fraser DJ. How well can captive breeding programs conserve biodiversity? A review of salmonids. Evolut Appl. 2008;1 (4):535-86.

14. Fisheries and Oceans Canada. Risks and benefits of juvenile to adult captive-reared supplementation activities to fitness of wild Atlantic salmon (Salmo salar). DFO Canadian Science Advisory Secretariat Science Advisory Report 2016/017. 2016.

15. Converse SJ, Moore CT, Folk MJ, Runge MC. A matter of tradeoffs: reintroduction as a multiple objective decision. J Wildl Manag. 2013;77(6):1145-56

16. Gum B, Lange M, Geist J. A critical reflection on the success of rearing and culturing juvenile freshwater mussels with a focus on the endangered freshwater pearl mussel (Margaritifera margaritifera L.). Aquat Conserv Mar Freshw Ecosyst. 2011;21:743-51.

17. IUCN. The IUCN Red List of threatened species. Version 2014.2. 2014 http://www.iucnredlist.org. ISSN 2307-8235. 
18. Collaboration for Environmental Evidence. Guidelines and Standards for Evidence synthesis in Environmental Management. Version 5.0. In: Pullin AS, Frampton GK, Livoreil B, Petrokofsky G, editors. 2018. http://www.envir onmentalevidence.org/information-for-authors. Accessed 15 Nov 2017.

19. Haddaway NR, Macura B, Whaley P, Pullin AS. ROSES Reporting standards for systematic evidence syntheses: pro forma, flow-diagram and descriptive summary of the plan and conduct of environmental systematic reviews and systematic maps. Environ Evid. 2018;7:7.

20. Government of Canada. Species at Risk Public Registry. https://www.regis trelep-sararegistry.gc.ca. Accessed 23 Nov 2017.

21. Social Science Research Unit, EPPI-Reviewer 4. UCL Institute of Education. 2016. https://eppi.ioe.ac.uk/. Accessed 12 July 2018.

22. Livoreil B, Glanville J, Haddaway NR, Bayliss H, Bethel A, Lachapelle FF, et al. Systematic searching for environmental evidence using multiple tools and sources. Environ Evid. 2017;6(1):23.
23. Waples RS, Ford MJ, Schmitt D. Empirical results of salmon supplementation in the Northeast Pacific: a preliminary assessment. In: Bert TM, editor. Ecological and genetic implications of aquaculture activities. Dordrecht: Springer; 2007. p. 383-403.

24. Frankham R, Ballou JD, Briscoe DA. Introduction to conservation genetics. Cambridge: Cambridge University Press. 2004:419-470. ISBN 9780521702713. OCLC 965796229.

25. Sciberras M, Jenkins SR, Kaiser MJ, Hawkins SJ, Pullin AS. Evaluating the biological effectiveness of fully and partially protected marine areas. Environ Evid. 2013;2(1):1-31.
Ready to submit your research? Choose BMC and benefit from:

- fast, convenient online submission

- thorough peer review by experienced researchers in your field

- rapid publication on acceptance

- support for research data, including large and complex data types

- gold Open Access which fosters wider collaboration and increased citations

- maximum visibility for your research: over 100M website views per year

At BMC, research is always in progress.

Learn more biomedcentral.com/submissions 